\title{
Two New Extensions of the Hales-Jewett Theorem
}

\author{
RANDAll McCutcheon* \\ Department of Mathematics \\ University of Maryland \\ College Park, MD 20742 \\ randall@math.umd.edu
}

Submitted: June 30, 2000; Accepted: September 28, 2000

\begin{abstract}
We prove two extensions of the Hales-Jewett coloring theorem. The first is a polynomial version of a finitary case of Furstenberg and Katznelson's multiparameter elaboration of a theorem, due to Carlson, about variable words. The second is an "idempotent" version of a result of Carlson and Simpson.
\end{abstract}

MSC2000: Primary 05D10; Secondary 22A15.

For $k, N \in \mathbf{N}$, let $\mathcal{W}_{k}^{N}$ denote the set of length- $N$ words on the alphabet $\{0,1, \cdots, k-$ $1\}$. A variable word over $\mathcal{W}_{k}^{N}$ is a word $w(x)$ of length $N$ on the alphabet $\{0,1, \cdots, k-$ $1, x\}$ in which the letter $x$ appears at least once. If $w(x)$ is a variable word and $i \in\{0,1, \ldots, k-1\}$, we denote by $w(i)$ the word that is obtained by replacing each occurrence of $x$ in $w(x)$ by an $i$. The Hales-Jewett theorem states that for every $k, r \in \mathbf{N}$, there exists $N=N(k, r) \in \mathbf{N}$ such that for any partition $\mathcal{W}_{k}^{N}=\bigcup_{i=1}^{r} C_{i}$, there exist $j, 1 \leq j \leq r$, and a variable word $w(x)$ over $\mathcal{W}_{k}^{N}$ such that $\{w(i): i \in$ $\{0,1, \ldots, k-1\}\} \subset C_{j}$.

\section{Finitary extensions.}

In [BL], V. Bergelson and A. Leibman provided a "polynomial" version of the HalesJewett theorem. In order to formulate their result, we must develop some terminology. Let $l \in \mathbf{N}$. A set-monomial (over $\mathbf{N}^{l}$ ) in the variable $X$ is an expression $m(X)=$ $S_{1} \times S_{2} \times \cdots \times S_{l}$, where for each $i, 1 \leq i \leq l, S_{i}$ is either the symbol $X$ or a nonempty singleton subset of $\mathbf{N}$ (these are called coordinate coefficients). The degree of the monomial is the number of times the symbol $X$ appears in the list $S_{1}, \cdots, S_{l}$. For example, taking $l=3, m(X)=\{5\} \times X \times X$ is a set-monomial of degree 2, while $m(X)=X \times\{17\} \times\{2\}$ is a set-monomial of degree 1 . A set-polynomial is an expression of the form $P(X)=m_{1}(X) \cup m_{2}(X) \cup \cdots \cup m_{k}(X)$, where $k \in \mathbf{N}$ and $m_{1}(X), \cdots, m_{k}(X)$ are set-monomials. The degree of a set-polynomial is the largest degree of its set-monomial "summands", and its constant term consists of the "sum" of

${ }^{*}$ The author acknowledges support from the National Science Foundation via a post doctoral fellowship administered by the University of Maryland. 
those $m_{i}$ that are constant, i.e. of degree zero. Finally, we say that two set polynomials are disjoint if they share no set-monomial summands in common.

Let $\mathcal{F}(S)$ denote the family of non-empty finite subsets of a set $S$. Any nonempty set polynomial $p(A)$ determines a function from $\mathcal{F}(\mathbf{N})$ to $\mathcal{F}\left(\mathbf{N}^{l}\right)$ in the obvious way (interpreting the symbol $\times$ as Cartesian product and the symbol $\cup$ as union). Notice that if $P(X)$ and $Q(X)$ are disjoint set-polynomials and $B \in \mathcal{F}(\mathbf{N})$ contains no coordinate coefficients of either $P$ or $Q$ then $P(B) \cap Q(B)=\emptyset$.

Here now is the Bergelson-Leibman coloring theorem.

Theorem 1.1. Let $l \in \mathbf{N}$ and let $\mathcal{P}$ be a finite family of set-polynomials over $\mathbf{N}^{l}$ whose constant terms are empty. Let $I \subset \mathbf{N}$ be any finite set and let $r \in \mathbf{N}$. There exists a finite set $S \subset \mathbf{N}$, with $S \cap I=\emptyset$, such that if $\mathcal{F}\left(\bigcup_{P \in \mathcal{P}} P(S)\right)=\bigcup_{i=1}^{r} C_{i}$ then there exists $i, 1 \leq i \leq r$, some non-empty $B \subset S$, and some $A \subset \bigcup_{P \in \mathcal{P}} P(S)$ with $A \cap P(B)=\emptyset$ for all $P \in \mathcal{P}$ and $\{A \cup P(B): P \in \mathcal{P}\} \subset C_{i}$.

Although the "polynomial" nature of Theorem 1.1 is at once clear, it is not immediately obvious that it includes the Hales-Jewett theorem as a special case, so we shall give a different formulation, and derive it from Theorem 1.1.

Let $k, N, d \in \mathbf{N}$. We denote by $\mathcal{M}_{k}^{N}(d)$ the set of all function $\phi:\{1,2, \ldots, N\}^{d} \rightarrow$ $\{0,1, \ldots, k-1\}$. When $d=2$, one may identify this with the set of $N \times N$ matrices with entries belonging to $\{0,1, \ldots, k-1\}$, so in general we shall refer to the members of $\mathcal{M}_{k}^{N}(d)$ as matrices, even when $d>2$. A variable matrix over $\mathcal{M}_{k}^{N}(d)$ is a function $\psi:\{1,2, \ldots, N\}^{d} \rightarrow\{0,1, \ldots, k-1, x\}$ for which $x$ appears in the range. The support of $\psi$ is the set $\psi^{-1}(x)$; that is, the set of locations in the matrix where the symbol $x$ appears. If $\psi$ is a variable matrix over $\mathcal{M}_{k}^{N}(d), \psi$ is said to be standard if its support has the form $B^{d}$ for some $B \subset\{1,2, \ldots, N\}$.

We shall also consider multi-variable matrices $\psi:\{1,2, \ldots, N\}\}^{d} \rightarrow\{0,1, \ldots, k-$ $\left.1, x_{1}, x_{2}, \ldots, x_{t}\right\}$. In this case we require that all the $x_{i}$ appear in the range, and we call $\psi^{-1}\left(x_{i}\right)$ the $i$ th support of $\psi$. If $\psi$ is a $t$-variable matrix then $\psi$ gives rise, via substitution, to a function $w\left(x_{1}, \ldots, x_{t}\right):\{0, \ldots, k-1\} \rightarrow \mathcal{M}_{k}^{N}(d)$, and we will often refer to this induced $w$ instead of to $\psi$ when dealing with variable matrices.

We require the following nonconventional notion of addition of matrices. We will introduce this notion in the context of dimension 2, although the obvious analogs are valid in arbitrary dimension. Let $w=\left(w_{i j}\right)_{i, j=1}^{M}$ and $y=\left(y_{i j}\right)_{i, j=1}^{M}$ be matrices (variable or otherwise). If there exist disjoint sets $W$ and $Y$, whose union is $\{1, \ldots, M\}^{2}$, such that $w_{i j}=0$ for $(i, j) \in W$ and $y_{i j}=0$ for $(i, j) \in Y$, then we define $w+y=\left(z_{i j}\right)_{i, j=1}^{M}$, where $z_{i j}=w_{i j}$ if $(i, j) \in Y$ and $z_{i j}=y_{i j}$ if $(i, j) \in W$. If however there exists $(i, j) \in\{1, \ldots, M\}^{2}$ such that $w_{i j} \neq 0 \neq y_{i j}$ then the sum $w+y$ is undefined.

Theorem 1.2 The following are equivalent:

(a) Theorem 1.1 .

(b) Let $d \in \mathbf{N}$ and let $\left(P_{i}(X)\right)_{i=1}^{t}$ be pairwise disjoint set-polynomials over $\mathbf{N}^{d}$ having empty constant term and let $\bar{J}$ be any finite subset of $\mathbf{N}$ containing all coordinate 
coefficients represented in the $P_{i}$ 's. Let $k, r \in \mathbf{N}$. There exists $N \in \mathbf{N}$ having the property that if $\mathcal{M}_{k}^{N}(d)=\bigcup_{i=1}^{r} C_{i}$ then there exists a set $B \subset\{1,2, \ldots, N\} \backslash J$, a variable matrix $w\left(x_{1}, \ldots, x_{t}\right)$, and $n$, with $1 \leq n \leq r$, such that

(i) The $i$ th support of $w_{i}$ is $P_{i}(B), 1 \leq i \leq t$,

(ii) $\left\{w\left(i_{1}, \ldots, i_{t}\right): i_{j} \in\{0,1, \ldots, k-1\}, 1 \leq j \leq t\right\} \subset C_{n}$, and

(iii) $w$ is 0 on $J^{d}$.

(c) Let $k, r, d \in \mathbf{N}$. There exists $N$ such that for every partition $\mathcal{M}_{k}^{N}(d)=\bigcup_{i=1}^{r} C_{i}$ there is a standard variable matrix $w(x)$ over $\mathcal{M}_{k}^{N}(d)$ such that $\{w(i): i \in\{0,1, \ldots, k-$ 1\}\} lies in one cell $C_{j}$.

Proof. First we show (a) implies (b). Choose $b \in \mathbf{N}$ with $2^{b} \geq k$ and consider the set

$$
\mathcal{P}=\left\{\bigcup_{s=1}^{t}\left(E_{s} \times P_{s}(X)\right): E_{s} \subset\{1, \ldots, b\}, 1 \leq s \leq t\right\}
$$

$\mathcal{P}$ is a finite family of set polynomials over $\mathbf{N}^{d+1}$. Let $I=J \cup\{1, \ldots, b\}$ and let $l=d+1$. Now pick a finite subset $S \subset \mathbf{N}$ as guaranteed by Theorem 1.1. Notice in particular that $S \cap I=\emptyset$. Pick $N \in \mathbf{N}$ such that $S \cup I \subset\{1, \ldots, N\}$. Suppose that $\mathcal{M}_{k}^{N}(d)=\bigcup_{i=1}^{r} C_{i}$. Form a map $\pi: \mathcal{F}\left(\{1, \ldots, b\} \times\{1, \ldots, N\}^{d}\right) \rightarrow \mathcal{M}_{k}^{N}(d)$ as follows:

$$
(\pi(A))\left(a_{1}, \ldots, a_{d}\right)=\min \left\{\sum_{\left(j, a_{1}, \ldots, a_{d}\right) \in A} 2^{j-1}, k-1\right\} .
$$

Now put $D_{i}=\pi^{-1}\left(C_{i}\right), 1 \leq i \leq r$. Then $\mathcal{F}\left(\bigcup_{P \in \mathcal{P}} P(S)\right) \subset \bigcup_{i=1}^{r} D_{i}$ so there exist $B \subset S$ and $A \subset \bigcup_{P \in \mathcal{P}} P(S)$ with $A \cap P(B)=\emptyset$ for all $P \in \mathcal{P}$ (in particular $\left.A \cap\left(\{1, \ldots, b\} \times P_{i}(B)\right)=\emptyset, 1 \leq i \leq t\right)$ and such that for some $z, 1 \leq z \leq r$,

$$
\left\{A \cup \bigcup_{s=1}^{t}\left(E_{s} \times P_{s}(B)\right): E_{s} \subset\{1, \ldots, b\}, 1 \leq s \leq t\right\} \subset D_{z} .
$$

Define a variable matrix $\psi=w\left(x_{1}, \ldots, x_{t}\right)$ over $\mathcal{M}_{k}^{N}(d)$ by

1. $\psi\left(\left(a_{1}, \ldots, a_{d}\right)\right)=x_{i}$ if $\left(a_{1}, \ldots, a_{d}\right) \in P_{i}(B)$, and

2. $\psi\left(\left(a_{1}, \ldots, a_{d}\right)\right)=\pi(A)\left(a_{1}, \ldots, a_{d}\right)$ otherwise.

(Recall that the sets $\left\{P_{i}(B): 1 \leq i \leq t\right\}$ are pairwise disjoint, owing to the fact that the $P_{i}$ 's are pairwise disjoint and $B$ contains no coordinate coefficients of any $P_{i}$.)

The $i$ th support of $w$ is clearly $P_{i}(B), 1 \leq i \leq t$. Now for any $i_{1}, \ldots, i_{t} \in$ $\{0,1, \ldots, k-1\}$, we pick sets $E_{s} \subset\{1, \ldots b\}$ such that $\sum_{n \in E_{s}} 2^{n-1}=i_{s}, 1 \leq s \leq t$, and note that

$$
w\left(i_{1}, \ldots, i_{t}\right)=\pi(A)+\sum_{s=1}^{t} \pi\left(E_{s} \times P_{s}(B)\right)=\pi\left(A \cup \bigcup_{s=1}^{t}\left(E_{s} \times P_{s}(B)\right)\right) \in C_{z} .
$$


Since $J \subset I, S \cap I=\emptyset$ and $A \subset \bigcup_{P \in \mathcal{P}} P(S)$, we have $A \cap\left(\{1, \ldots, b\} \times J^{d}\right)=\emptyset$, so that $w$ is zero on $J^{d}$.

This finishes the proof that (a) implies (b). Letting $t=1$ and $P_{1}(X)=X^{d}$, one sees that (b) implies (c). Therefore all that remains is to show (c) implies (a).

Let $\left\{Q_{1}, \cdots, Q_{t}\right\}$ be the family of all set-monomials that appear in any of the set-polynomials of $\mathcal{P}$, and write $Q_{i}(X)=S_{1}^{(i)} \times \cdots \times S_{d}^{(i)}$, where each $S_{j}^{(i)}$ is either a singleton or the symbol $X$. Let $k=2^{t}$ and put $d=l$.

Let $N$ be as promised by (c) and choose $y \in \mathbf{N}$ larger than all coordinate coefficients in question and larger than any member of $I$. Set $S=\{y+1, \ldots, y+N\}$. Suppose now that $\mathcal{F}\left(\bigcup_{P \in \mathcal{P}} P(S)\right)=\bigcup_{i=1}^{r} C_{i}$.

Let $Y$ be the family of $t$-tuples of subsets of $\{1, \ldots, N\}^{d}$. We identify $Y$ with $\mathcal{M}_{k}^{N}(d)$ by

$$
\left(A_{1}, \ldots, A_{t}\right) \leftrightarrow w \text { if and only if } w\left(i_{1}, \ldots, i_{d}\right)=\sum_{s=1}^{t} 2^{1_{A_{s}}\left(\left(i_{1}, \ldots, i_{d}\right)\right)} .
$$

Our next task is to construct a map $\pi$ sending $Y$ (and thus, effectively, $\mathcal{M}_{k}^{N}(d)$ ) to $\mathcal{F}\left(\bigcup_{s=1}^{t} Q_{s}(S)\right)=\mathcal{F}\left(\bigcup_{P \in \mathcal{P}} P(S)\right)$. First we define $\pi$ for $t$-tuples of sets, one of which is a singleton and the rest of which are empty. Suppose then that $i$ is fixed, $A_{j}=\emptyset$ for $i \neq j$ and $A_{i}=\left\{\left(a_{1}, \ldots, a_{d}\right)\right\}$. Recall that $Q_{i}(X)=S_{1}^{(i)} \times \cdots S_{d}^{(i)}$, where some of the $S_{j}^{(i)}$ are singletons and some are $X$. Let $T=\left\{j: S_{j}^{(i)}=X\right\}$. Suppose that for all $j \in\{1, \ldots, d\} \backslash T, a_{j}=\min \left\{a_{i}: i \in T\right\}$. If this condition is not met, we set $\pi\left(\left(A_{1}, \cdots, A_{t}\right)\right)=\emptyset$. If the condition is met, put $b_{j}=S_{j}^{(i)}$ if $S_{j}^{(i)}$ is a singleton and $b_{j}=a_{j}+y$ if $S_{j}^{(i)}=X, 1 \leq j \leq d$, and set $\pi\left(A_{1}, \ldots, A_{t}\right)=\left\{\left(b_{1}, \ldots, b_{d}\right)\right\}$. We now extend $\pi$ to the desired domain by requiring that $\pi\left(A_{1} \cup B_{1}, \ldots, A_{t} \cup B_{t}\right)=$ $\pi\left(A_{1}, \ldots, A_{t}\right) \cup \pi\left(B_{1}, \ldots, B_{t}\right)$. (This extension is unique.)

We now confirm that $\pi$ has the following two properties. First, if $C \subset\{1, \ldots, N\}$, then letting $B=C+y=\{c+y: c \in C\}$, fixing $i$ and putting $A_{i}=C^{d}$ and $A_{j}=\emptyset$ for all $j \neq i, \pi\left(A_{1}, \ldots, A_{t}\right)=Q_{i}(B)$. Second, if $A_{i} \cap B_{i}=\emptyset$ for all $i$, $\pi\left(\left(A_{1}, \ldots, A_{t}\right)\right) \cap \pi\left(\left(B_{1}, \ldots, B_{t}\right)\right)=\emptyset$.

We now use the map $\pi$ to draw back the partition. Namely, let $D_{i}=\pi^{-1}\left(C_{i}\right)$, $1 \leq i \leq r$. Then $Y=\bigcup_{i=1}^{r} D_{i}$. But $Y$ is identified with $\mathcal{M}_{k}^{N}(d)$, so by (c) there exists a standard variable matrix $w(x)$ and some $z, 1 \leq z \leq r$, such that $W=\{w(i): i \in$ $\{0,1, \ldots, k-1\}\} \subset D_{z}$. (After the identification, of course.)

Let $C^{d}$ be the support of $w(x)$. Let $\left(A_{1}, \ldots, A_{t}\right)$ be the member of $Y$ that is identified with $w(0)$. Then $A_{i} \cap C^{d}=\emptyset$ for $1 \leq i \leq t$, so that $\pi\left(\left(A_{1}, \ldots, A_{t}\right)\right) \cap$ $\pi\left(\left(C^{d}, \ldots, C^{d}\right)\right)=\emptyset$. Moreover, in $Y, W$ takes the form

$$
W=\left\{\left(A_{1}, \ldots, A_{t}\right) \cup\left(F_{1}, \ldots, F_{t}\right): F_{i} \in\left\{\emptyset, C^{d}\right\}, 1 \leq i \leq t\right\} .
$$

Let $A=\pi\left(\left(A_{1}, \ldots, A_{t}\right)\right)$ and let $B=C+y$. Let $P \in \mathcal{P}$ and choose a set $E \subset\{1, \ldots, t\}$ such that $P(X)=\bigcup_{i \in E} Q_{i}(X)$. Next put $F_{j}=C^{d}$ if $j \in E$ and $F_{j}=\emptyset$ otherwise. Then $\left(A_{1}, \ldots, A_{t}\right) \cup\left(F_{1}, \ldots, F_{t}\right) \in W$. But $\pi(W) \subset C_{z}$, so $\left(A \cup \bigcup_{i \in E} Q_{i}\left(B^{d}\right)\right) \in C_{z}$. 
Formulations (a) and (b) in Theorem 1.2 are more powerful, on the surface, than formulation (c) and hence it is good to have them on hand for some applications, but formulation (c) has aesthetic advantages. For one, when $d=1$ it gives precisely the Hales-Jewett theorem.

We now shift our focus slightly. Let $A$ be a finite field and let $n \in \mathbf{N}$. Then $A^{n}$ is a vector space over $A$. A translate of a $t$-dimensional vector subspace of $A^{n}$ is called a $t$-space. The following theorem was proved by Graham, Leeb and Rothschild ([GLR]).

Theorem 1.3 Let $r, n, t \in \mathbf{N}$. There exists $N=N(r, n, t)$ such that for any $r$-coloring of the $n$-spaces of $A^{N}$ there exists a $t$-space $V$ such that the family of $n$-spaces contained in $V$ is monochromatic.

We mention this result because it is so well known. It is not quite in keeping with our theme, namely extensions of the Hales-Jewett theorem, but if we restrict attention to a certain sub-class of $n$-spaces, the situation becomes much more "Hales-Jewettlike".

Recall that a variable word over $\mathcal{W}_{k}$ is a word on the alphabet $\{1,2, \cdots, k, x\}$ in which the symbol $x$ appears at least once. An $n$-variable word is a word on the alphabet $\left\{1, \cdots, k, x_{1}, \cdots, x_{n}\right\}$ in which all the $x_{i}$ 's occur and for which no occurrence of $x_{i+1}$ precedes an occurrence of $x_{i}, 1 \leq i \leq n-1$. If $w\left(x_{1}, \cdots, x_{n}\right)$ is an $n$-variable word over $\mathcal{W}_{k}^{M}$ then the set $\left\{w\left(t_{1}, t_{2}, \cdots, t_{n}\right): 1 \leq t_{i} \leq k, i=1, \cdots, n\right\}$ will be called the space associated with $w$. (Notice now that if $k=p^{s}$ for some prime $p$ and $s \in \mathbf{N}$ and we identify $\{0,1, \ldots, k-1\}$ with a field $A$ having $p^{s}$ elements, choose a basis $\left\{v_{1}, \cdots, v_{M}\right\}$ for $A^{M}$ and identify the word $w_{1} w_{2} \cdots w_{M}$ with the vector $\sum_{i=1}^{M} w_{i} v_{i}$, then the space associated with an $n$-variable word is indeed an $n$-space in $A^{N}$. However, not all $n$-spaces can be obtained in this way.)

If $w$ is a $t$-variable word and $v$ is an $n$-variable word and the space associated with $v$ is contained in the space associated with $w, v$ will be called an $n$-subword of $w$. Another way of seeing this is, if $w\left(y_{1}, \cdots, y_{t}\right)$ is a $t$-variable word then the $n$-variable subwords of it (in the variables $\left.x_{1}, \cdots, x_{n}\right)$ are of the form $w\left(z_{1}, \cdots, z_{t}\right.$ ), where $z_{1} \cdots z_{t}$ is an $n$-variable word over $\mathcal{W}_{k}(t)$.

The following theorem is a finitary consequence of a generalization of T. Carlson's theorem ([C, Lemma 5.9]) due to H. Furstenberg and Y. Katznelson (see [FK, Theorem 3.1]). It extends the Hales-Jewett theorem in the following sense. If we call regular words (that is, elements of $\mathcal{W}_{k}^{M}$ ) 0 -variable words, then the Hales-Jewett theorem corresponds to the case $n=0, t=1$ of Theorem 1.4 .

Theorem 1.4 Let $k, r, n, t \in \mathbf{N}$ be given. There exists $M=M(k, r, n, t)$ such that for every $r$-cell partition of the $n$-variable words over $\mathcal{W}_{k}^{M}$ there exists a $t$-variable word all of whose $n$-subwords lie in the same cell.

We seek now to give a polynomial analog of Theorem 1.4. To this end, let $k, N, d, n \in \mathbf{N}$ and suppose we have non-empty sets $B_{i} \subset\{1, \ldots, N\}, 1 \leq i \leq n$, 
with $B_{1}<\cdots<B_{n}$. (Here and elsewhere in this paper, we write $A<B$ where $A$ and $B$ are non-empty finite subsets of $\mathbf{N}$ when $a<b$ for all $a \in A$ and $b \in B$.) If $w\left(x_{1}, \cdots, x_{n^{d}}\right)$ is an $n^{d}$-variable matrix over $\mathcal{M}_{k}^{N}(d)$ whose supports are the sets $B_{i_{1}} \times B_{i_{2}} \times \cdots \times B_{i_{d}}, 1 \leq i_{1}, \ldots, i_{d} \leq n$, then $w$ is said to be a standard $n^{d}$-variable matrix. The space associated with $w$ is $\left\{w\left(i_{1}, \ldots, i_{n^{d}}\right): i_{1}, \ldots, i_{n^{d}} \in\{0,1, \ldots, k-1\}\right\}$.

If $n_{1} \leq n_{2}, w_{1}$ is a standard $n_{1}^{d}$-variable matrix, $w_{2}$ is a standard $n_{2}^{d}$-variable matrix, and the space associated with $w_{1}$ is contained in the space associated with $w_{2}$, then we will say that $w_{1}$ is a submatrix of $w_{2}$.

Our main theorem in this section is Theorem 1.7. This theorem will be a version of Theorem 1.4 valid in any finite dimension $d$. However, in order to simplify the proof notationally, we will take $d$ to be 2 . We need two lemmas for the proof.

Lemma 1.5 Let $R, k, T \in \mathbf{N}$. There exists $M=M(R, k, T) \in \mathbf{N}$ having the following property: Let $\mathcal{E}$ denote the set of matrices $\left(a_{i j}\right)_{i, j=1}^{T+M}$ such that

(a) $\left(a_{i j}\right)_{i, j=1}^{T+M}$ is a standard $n^{2}$-variable matrix, and

(b) $a_{i j} \in\{0,1, \ldots, k-1\}$ if either $i>T$ or $j>T$ (that is, all the supports of $\left(a_{i j}\right)_{i, j=1}^{T+M}$ lie in $\left.\{1, \ldots, T\}^{2}\right)$.

Then for any $R$-coloring $\gamma$ of $\mathcal{E}$ there exists a $(2 T+1)$-variable matrix $w\left(x_{1}, \ldots, x_{2 T+1}\right)$ $=\left(b_{i j}\right)_{i, j=1}^{T+M}$ over $\mathcal{M}_{k}^{T+M}(2)$ that satisfies:

(1) $b_{i j}=0$ if $(i, j) \in\{1, \ldots, T\}^{2}$.

(2) There exists a non-empty set $B \subset\{T+1, \ldots, T+M\}$ such that the supports of $w$ are $\{i\} \times B$ and $B \times\{i\}, i \in\{1, \ldots, T\}, B \times B$.

(3) For any standard $n^{2}$-variable matrix $m=\left(c_{i j}\right)_{i, j=1}^{T+M}$ satisfying $c_{i j}=0$ if $(i, j) \notin$ $\{1, \ldots, T\}^{2}$, the set $\left\{m+w\left(i_{1}, \ldots, i_{2 T+1}\right): i_{j} \in\{0,1, \ldots, k-1\}, 1 \leq j \leq 2 T+1\right\}$ is $\gamma$-monochromatic.

Proof. Let $P_{i}(X), 1 \leq i \leq 2 T+1$, denote the set polynomials $\{i\} \times X$ and $X \times\{i\}$, $i \in\{1, \ldots, T\}$, and $X \times X$. These are pairwise disjoint set-polynomials (in fact, distinct set-monomials). Let $\mathcal{G}$ be the set of all standard $n^{2}$-variable matrices over $\mathcal{M}_{k}^{T}(2)$. Let $J=\{1, \ldots, T\}, t=2 T+1, r=R^{|\mathcal{G}|}+1, d=2$, and put $M=N-T$, where $N$ is the number guaranteed by Theorem 1.2 (b). Let $\gamma$ be an $R$-coloring of $\mathcal{E}$.

We now construct a $\left(R^{|\mathcal{G}|}+1\right)$-cell partition of $\mathcal{M}_{k}^{N}(2)$. For $\left(d_{i j}\right)_{i, j=1}^{N},\left(f_{i j}\right)_{i, j=1}^{N} \in$ $\mathcal{M}_{k}^{N}(2)$, we write $\left(d_{i j}\right)_{i, j=1}^{N} \sim\left(f_{i j}\right)_{i, j=1}^{N}$ if for every standard $n^{2}$-variable matrix $m=$ $\left(e_{i j}\right)_{i, j=1}^{T+M}$ satisfying $e_{i j}=0$ for all $(i, j) \notin\{1, \ldots, T\}^{2}$, we have $\gamma\left(m+\left(d_{i j}\right)_{i, j=1}^{N}\right)=$ $\gamma\left(m+\left(f_{i j}\right)_{i, j=1}^{N}\right)$, in the sense that if either side of this expression is defined then so is the other and they are equal. (Hence in particular all matrices that have a non-zero entry for any index point in $\{1, \ldots, T\}^{2}$ are relegated to the same equivalence class. The other equivalence classes are characterized by the value of $\gamma$ at $|\mathcal{G}|$ points, hence the equivalence classes of $\sim$ form an $r$-cell partition.)

According to the conditions whereby $M$ was chosen, there exists a non-empty set $B \subset\{1, \ldots, N\} \backslash J=\{T+1, \ldots, T+M\}$ and a variable matrix $w\left(x_{1}, \ldots, x_{2 T+1}\right)=$ $\left(b_{i j}\right)_{i, j=1}^{T+M}$ such that the supports of $w$ are $P_{i}(B), 1 \leq i \leq 2 T+1$, and the set 
$\left\{w\left(i_{1}, \ldots, i_{2 T+1}\right): i_{j} \in\{0,1, \ldots, k-1\}, 1 \leq j \leq 2 T+1\right\}$ lies entirely in a single equivalence class of $\sim$ and such that moreover $b_{i j}=0$ for all $(i, j) \in J^{2}=\{1, \ldots, T\}^{2}$. The variable matrix thus chosen satisfies (1), (2) and (3).

Our second lemma is a finitary version of a theorem proved independently by Milliken ([Mi] ) and Taylor ([T]). Recall that if $A$ is a set then $\mathcal{F}(A)$ is the family of nonempty finite subsets of $A$. We write $\mathcal{F}=\mathcal{F}(\mathbf{N})$ as a kind of shorthand. Recall that for $\alpha, \beta \in \mathcal{F}$, we write $\alpha<\beta$ if $\max \alpha<\min \beta$. For $k \in \mathbf{N}$, and a sequence $\left(\alpha_{i}\right)_{i=1}^{\infty} \subset \mathcal{F}$, we write $F U\left(<\alpha_{i}>_{i=1}^{\infty}\right)=\left\{\bigcup_{i \in A} \alpha_{i}: A \in \mathcal{F}\right\}$. (FU stands for "finite unions." One may consider the set of finite unions of a finite sequence as well, of course.) If $\mathcal{G} \subset \mathcal{F}$, let $\mathcal{G}_{<}^{k}$ be the set of $k$-tuples $\left(\alpha_{1}, \ldots, \alpha_{k}\right)$ in $\mathcal{G}^{k}$ for which $\alpha_{1}<\alpha_{2}<\cdots \alpha_{k}$. The Milliken-Taylor theorem states that for any finite partition $\mathcal{F}_{<}^{k}=\bigcup_{i=1}^{r} C_{i}$, there exists $j$, with $1 \leq j \leq r$, and a sequence $\left(\alpha_{i}\right)_{i=1}^{\infty}$, with $\alpha_{1}<\alpha_{2}<\cdots$, such that $\left(F U\left(<\alpha_{i}>_{i=1}^{\infty}\right)\right)_{<}^{k} \subset C_{j}$.

We shall not need the full strength of the Milliken-Taylor theorem, but only the following finitary version of it.

Lemma 1.6 Let $r, n, t \in \mathbf{N}$. There exists $L=L(r, n, t) \in \mathbf{N}$ such that if $\left\{\left(\alpha, \ldots, \alpha_{n}\right)\right.$ : $\left.\emptyset \neq \alpha_{i} \subset\{1, \ldots, L\}, \alpha_{1}<\alpha_{2}<\cdots<\alpha_{n}\right\}=\bigcup_{i=1}^{r} C_{i}$ then there exist non-empty sets $\alpha_{i} \subset\{1, \ldots, L\}, 1 \leq i \leq t$, with $\alpha_{1}<\alpha_{2}<\cdots<\alpha_{t}$, and $j, 1 \leq j \leq r$, with $\left(F U\left(<\alpha_{i}>_{i=1}^{t}\right)\right)_{<}^{n} \subset C_{j}$.

Here now is the main theorem of this section.

Theorem 1.7 Let $k, r, n, t, d \in \mathbf{N}$. There exists $N=N(k, r, n, t, d)$ such that for every $r$-cell partition of the standard $n^{d}$-variable matrices over $\mathcal{M}_{k}^{N}(d)$, there exists a standard $t^{d}$-variable matrix over $\mathcal{M}_{k}^{N}(d)$ all of whose standard $n^{d}$-variable submatrices lie in the same cell.

Before giving the proof of Theorem 1.7, let us make a few remarks about notation and also Lemma 1.5. First, the object $\mathcal{E}$ defined in the lemma consists of variable words with supports in $\{1, \ldots, T\}^{2}$, and the variable word that is found must have zero entries over $\{1, \ldots, T\}^{2}$. We note that there is nothing remarkable here about the set $\{1, \ldots, T\}^{2}$. Once $M$ has been chosen, any set $S^{2} \subset\{1, \ldots, M+T\}^{2}$ where $|S|=T$, would serve just as nicely in this capacity. This is a simple result of the fact that standard variable matrices remain such upon permuting the indices $\{1, \ldots, M+T\}$.

Next, the lemma as stated applies to $\mathcal{M}_{k}^{T+M}(2)$ and variable words over it. In our application of it, we shall be applying it in the context of an isomorphic copy of $\mathcal{M}_{k}^{T+M}$, namely the space determined by an appropriate standard $(M+T)^{2}$-variable matrix. Notationally, it is convenient to write such a variable matrix with a matrix of variables, namely as $w\left(\left(x_{i j}\right)_{i, j=1}^{T+M}\right)$, where it is understood that the variable $x_{i j}$ has support $B_{i} \times B_{j}$ for some non-empty sets $B_{1}<B_{2}<\cdots<B_{T+M}$. When applying Lemma 1.6 to the space associated with the variable matrix, it is important to note that if $\left(m_{i j}\right)$ is a standard $n^{2}$-variable matrix over $\mathcal{M}_{k}^{T+M}(2)$, then $w\left(\left(m_{i j}\right)_{i, j=1}^{T+M}\right)$ 
becomes, upon substitution, a standard $n^{2}$-variable matrix. Moreover, all standard $n^{2}$-variable matrices over the space in question arise in this fashion.

Proof of Theorem 1.7 Recall that our plan is to confine ourselves in the proof to the $d=2$ case. The changes necessary to extend the proof to general $d$ are minor and rather obvious, but it will be difficult enough to keep track of all the symbols in two dimensions, so we opt to simplify.

Let $L=L(r, n, t)$ be as guaranteed by Lemma 1.6. We now use Lemma 1.5 iteratively. Let $M_{1}=M(r, k, L-1)$. Having chosen $M_{1}, \ldots, M_{s-1}$, let $M_{s}=M(r, k, L-$ $\left.s+M_{1}+M_{2}+\cdots+M_{s-1}\right)$. Continue until $M_{L}=M\left(r, k, M_{1}+\cdots+M_{L-1}\right)$ has been chosen. For $i=1,2, \ldots, L$, let $N_{i}=M_{1}+\cdots+M_{i}$, and put $N=N_{L}$.

Suppose now we are given an $r$-coloring $\gamma$ of the standard $n^{2}$-variable matrices over $\mathcal{M}_{k}^{N}(2)$. By virtue of the way $M_{L}$ was chosen, we can find a non-empty set $B_{L} \subset\left\{N_{L-1}+1, \ldots, N_{L}\right\}$ and a $\left(2 N_{L-1}+1\right)$-variable matrix $W_{L}$ that has zero entries on $\left\{1, \ldots, N_{L-1}\right\}^{2}$ and whose supports are $\{i\} \times B_{L}$ and $B_{L} \times\{i\}, 1 \leq i \leq N_{L-1}$, and $B_{L} \times B_{L}$, with the following property: for every standard $n^{2}$-variable matrix $m$ over $\mathcal{M}_{k}^{N}(2)$ whose entries are zero except possibly on $\left\{1, \ldots, N_{L-1}\right\}^{2}$, the value of $\gamma$ on $m+w_{L}\left(i_{1}, \cdots, i_{2 N_{L-1}+1}\right)$ remains constant as the $i_{j}$ 's move independently over $\{0,1, \ldots, k-1\}$.

We now restrict attention to the space, call it $\mathcal{S}_{L-1}$, of matrices $p+f$, where $p$ has zero entries except possibly on $\left\{1, \ldots, N_{L-1}\right\}^{2}$ and $f$ is in the range of $w_{L}$. This space may be realized as the space associated with an appropriately chosen standard $\left(N_{L-1}+1\right)^{2}$-variable matrix, hence is isomorphic to $\mathcal{M}_{k}^{N_{L-1}+1}$, and so the remarks made prior to the proof apply. Namely, we can use Lemma 1.5 in this space. Specifically, since $M_{L-1}=M\left(r, k, N_{L-2}+1\right)$, we can find a non-empty set $B_{L-1} \subset\left\{N_{L-2}+1, \ldots, N_{L-1}\right\}$ and a variable matrix $w_{L-1}\left(x_{1}, \ldots, x_{2\left(N_{L-1}+1\right)+1}\right)$ over $\mathcal{S}_{L-1}$ with the following properties. (This part is somewhat tedious, as one must be very careful to interpret Lemma 1.5 correctly in this specialized context of a space that is merely isomorphic to $\mathcal{M}_{k}^{N}(2)$.)

1. Let $w_{L-1}=\left(b_{i j}\right)$ and let $w_{L}=\left(c_{i j}\right)$. If $(i, j) \notin\left\{1, \ldots, N_{L-1}\right\}^{2}$, and $c_{i j} \in$ $\{0,1, \ldots, k-1\}$, then $b_{i j}=c_{i j}$.

2. $b_{i j}=0$ for all $(i, j) \in\left(\left\{1, \ldots, N_{L-2}\right\} \cup B_{L}\right)^{2}$.

3. The supports of $w_{L-1}$ are the sets $\{i\} \times B_{L-1}$ and $B_{L-1} \times\{i\}, i \in\left\{1, \ldots, N_{L-2}\right\}$, $B_{L} \times B_{L-1}, B_{L-1} \times B_{L}$, and $B_{L-1} \times B_{L-1}$.

4. Let $m=\left(d_{i j}\right)$ be any standard $n^{2}$-variable matrix such that $d_{i j}=0$ for every $(i, j) \notin\left(\left\{1, \ldots, N_{L-2}\right\} \cup B_{L}\right)^{2}$. Then the value of $\gamma$ remains constant on $m+$ $w_{L-1}\left(i_{1}, \ldots, i_{2 N_{L-1}+3}\right)$ as the $i_{j}$ 's run over $\{0,1, \ldots, k-1\}$ independently.

At the next stage we restrict attention to the space, call it $\mathcal{S}_{L-2}$, of matrices of the form $p+f$ where $f$ is in the range of $w_{L-1}$ and $p$ is constant on each of the sets:

a. $\{(i, j)\},(i, j) \in\left\{1, \ldots, N_{L-2}\right\}^{2}$,

b. $\{i\} \times B_{L}$ and $B_{L} \times\{i\}, i \in\left\{1, \ldots, N_{L-2}\right\}$,

c. $B_{L} \times B_{L}$, 
while being zero elsewhere.

This space is isomorphic to $\mathcal{M}_{k}^{N_{l-2}+2}$, and so by the way $M_{L-2}$ was picked, Lemma 1.5 applies. The variable word (over $\mathcal{S}_{L-2}$ ) $w_{L-2}$ that is found will have $2 N_{L-3}+5$ variables and its supports will be $\{i\} \times B_{L-2}$ and $B_{L-2} \times\{i\}$ for $i \in\left\{1, \ldots, N_{L-3}\right\}$, $B_{L-1} \times B_{L-2}, B_{L-2} \times B_{L-1}, B_{L} \times B_{L-2}, B_{L-2} \times B_{L}$, and $B_{L-2} \times B_{L-2}$. Here $\emptyset \neq B_{L-2} \subset\left\{N_{L-3}+1, \ldots, N_{L-2}\right\} . w_{L-2}$ will have zero entries in $\left(\left\{1, \ldots, N_{L-3}\right\} \cup\right.$ $\left.B_{L} \cup B_{L-1}\right)^{2}$. $w_{L-2}$ will agree with $w_{L-1}$ on those indices $(i, j)$ lying outside of $\left\{1, \ldots, N_{L-2}\right\}^{2}$ on which $w_{L-1}$ takes a value in $\{0,1, \ldots, k-1\}$. Finally if $m=\left(d_{i j}\right)$ is any standard $n^{2}$-variable matrix such that $d_{i j}=0$ for every $(i, j) \notin\left(\left\{1, \ldots, N_{L-3}\right\} \cup\right.$ $\left.B_{L} \cup B_{L-1}\right)^{2}$, then the value of $\gamma$ remains constant on $m+w_{L-2}\left(i_{1}, \ldots, i_{2 N_{L-2}+5}\right)$ as the $i_{j}$ 's run over $\{0,1, \ldots, k-1\}$ independently.

Continue choosing sets $B_{i}$ and variable matrices $w_{i}$. By the time $w_{1}$ is chosen, it's supports will be on $B_{i} \times B_{1}$ and $B_{1} \times B_{i}, 2 \leq i \leq L$, and $B_{1} \times B_{1}$, where $B_{1} \subset\left\{1, \ldots N_{1}\right\}$. $w_{1}$ will have zero entries on $B_{i} \times B_{j}, 2 \leq i, j \leq L$, and will agree with $w_{2}$ elsewhere (that is, on the entries of $w_{2}$ that are in $\{0,1, \ldots, k-1\}$ ) outside of $\left\{1, \ldots, N_{1}\right\}^{2} . w_{1}$ will have the property that for every standard $n^{2}$-variable matrix $m$, whose entries are constant over each set $B_{i} \times B_{j}, 2 \leq i, j \leq L$, and zero elsewhere, the value of $\gamma$ on $m+w_{1}\left(i_{1}, \ldots, i_{2 L+1}\right)$ remains constant as the $i_{j}$ 's move independently over $\{0,1, \ldots, k-1\}$.

Finally, let $v\left(\left(x_{i j}\right)_{i, j=1}^{L}\right)$ be the standard $L^{2}$-variable matrix that agrees with $w_{1}$ for those indices on which $w_{1}$ takes a value in $\{0,1, \ldots, k-1\}$, and whose variables $x_{i j}$ have supports $B_{i} \times B_{j}$, respectively, $1 \leq i, j \leq L$. The construction we have followed gives $v$ the following property: if $\left(h_{i j}\right)_{i, j=1}^{L}$ and $\left(s_{i j}\right)_{i, j=1}^{L}$ are standard $n^{2}$-variable matrices whose supports are identical, then $\gamma\left(v\left(\left(h_{i j}\right)_{i, j=1}^{L}\right)\right)=\gamma\left(v\left(\left(s_{i j}\right)_{i, j=1}^{L}\right)\right)$. In demonstrating this, we may assume without loss of generality that the two $L \times L$ matrices in question differ at only one entry, say at position $(x, y)$. Clearly $h_{x y}$ and $s_{x y}$ are in $\{0,1, \ldots, k-1\}$.

Suppose for convenience that $x \leq y$. One may show that there exist matrices $p_{1}, p_{2}$ and $m=\left(d_{i j}\right)$ such that

1. $p_{1}$ and $p_{2}$ are each in the range of $w_{x}$.

2. $m$ is a standard $n^{2}$-variable matrix with $d_{i j}=0$ if $(i, j) \notin\left(\left\{1, \ldots N_{x-1}\right\} \cup B_{L} \cup\right.$ $\left.B_{L-1} \cup \cdots \cup B_{x+1}\right)^{2}$.

3. $m+p_{1}=v\left(\left(h_{i j}\right)_{i, j=1}^{L}\right)$ and $m+p_{2}=v\left(\left(s_{i j}\right)_{i, j=1}^{L}\right)$.

Indeed, put $U=\left(\left\{1, \ldots N_{x-1}\right\} \cup B_{L} \cup B_{L-1} \cup \cdots \cup B_{x+1}\right)^{2}$. Let $m$ coincide with $v\left(\left(h_{i j}\right)_{i, j=1}^{L}\right)$ on $U$ and have zero entries on $U^{c}$, then let $p_{1}$ coincide with $v\left(\left(h_{i j}\right)_{i, j=1}^{L}\right)$ on $U^{c}$, and have zero entries on $U . p_{2}$ is chosen similarly, but with respect to $v\left(\left(s_{i j}\right)_{i, j=1}^{L}\right)$.

According to the criteria by which $w_{x}$ was chosen, $\gamma\left(m+p_{1}\right)=\gamma\left(m+p_{2}\right)$, as required.

Let us take stock of the situation. We have found a standard $L^{2}$-variable ma- 
trix $v$ with the property that the value of $\gamma$ on its standard $n^{2}$-variable sub-matrices $v\left(\left(h_{i j}\right)_{i, j=1}^{L}\right)$ depends only on the location of the supports of the variables in the underlying matrix $\left(h_{i j}\right)_{i, j=1}^{L}$. Now, these variables are always supported on sets $A_{i} \times A_{j}$, $1 \leq i, j \leq n$, where each $A_{i} \subset\{1, \ldots, L\}$ is non-empty and $A_{1}<A_{2}<\cdots<A_{n}$. In other words, the function $\gamma$ restricted to the standard $n^{2}$-variable submatrices of $v$ is the lift of an $r$-coloring $\gamma^{\prime}$ of the set $(\mathcal{F}(\{1, \ldots, L\}))_{<}^{n}$. By the choice of $L$, there thus exist non-empty sets $C_{i} \subset\{1, \ldots, L\}, 1 \leq i \leq t$, with $C_{1}<C_{2}<\cdots<C_{t}$, such that $\gamma^{\prime}$ is constant on the family of $n$-tuples $\left(A_{1}, \cdots, A_{n}\right)$, where $A_{i} \in F U\left(\left\{C_{1}, \ldots, C_{t}\right\}\right)$, $1 \leq i \leq n$, and $A_{1}<A_{2}<\cdots<A_{n}$. Let now $\left(h_{i j}\right)_{i, j=1}^{L}$ be any standard $t^{2}$-variable matrix over $\mathcal{M}_{k}^{L}(2)$ whose supports lie on $C_{i} \times C_{j}, 1 \leq i \leq t$. Then $v\left(\left(h_{i j}\right)_{i, j=1}^{L}\right)$ is a standard $t^{2}$-variable matrix over $\mathcal{M}_{k}^{N}(2)$ whose standard $n^{2}$-variable submatrices are $\gamma$-monochromatic.

Theorem 1.7 extends the Bergelson-Leibman coloring theorem in the sense that if one defines zero-variable matrices to be matrices with entries in $\{0,1, \ldots, k-1\}$ then Theorem 1.2 (c) is precisely the case $n=0, t=1$ of Theorem 1.7.

\section{Infinitary extensions.}

Let $k \in \mathbf{N}$ and let $w(x)$ be a variable word over $\mathcal{W}_{k}$. If the first letter of $w(x)$ is $x$, then we say that $w(x)$ is a left-sided variable word. The following "infinitary" Hales-Jewett theorem is due to T. Carlson and S. Simpson.

Theorem 2.1 ([CS]) Let $k, r \in \mathbf{N}$ and suppose $\mathcal{W}_{k}=\bigcup_{i=1}^{r} C_{i}$. Then there exists $z$, with $1 \leq z \leq r$, a variable word $w_{1}(x)$, and a sequence of left-sided variable words $\left(w_{i}(x)\right)_{i=2}^{\infty}$ such that for all $N \in \mathbf{N}$ and all $i_{1}, \cdots, i_{N} \in\{0,1, \ldots, k-1\}$, $w_{1}\left(i_{1}\right) w_{2}\left(i_{2}\right) \cdots w_{N}\left(i_{N}\right) \in C_{z}$.

Furstenberg and Katznelson indicated a similar theorem (see the remark following Theorem 2.5 in $[\mathrm{FK}])$.

Theorem 2.2 Let $k, r \in \mathbf{N}$ and suppose $\mathcal{W}_{k}=\bigcup_{i=1}^{r} C_{i}$. Then there exists $z$, with $1 \leq z \leq r$, and a sequence of variable words $\left(w_{i}(x)\right)_{i=1}^{\infty}$ such that for all $N \in \mathbf{N}$, all $b_{1}, b_{2}, \ldots, b_{N} \in \mathbf{N}$ with $b_{1}<b_{2}<\cdots<b_{N}$, and all $i_{1}, \cdots, i_{N} \in\{0,1, \ldots, k-1\}$, $w_{b_{1}}\left(i_{1}\right) w_{b_{2}}\left(i_{2}\right) \cdots w_{b_{N}}\left(i_{N}\right) \in C_{z}$.

Theorem 2.2 is stronger in the sense that one gets more products in the desired cell, but Theorem 2.1 is stronger in the sense that the variable words, excepting the first one, are required to be left-sided. One aesthetic advantage of left variable words is that the determination of the words becomes somewhat more canonical. So, for example, if one were given that $w_{1}(2) w_{2}(1)=225612114$ and $w_{1}(1) w_{2}(2)=125622124$, where $w_{2}(x)$ is known to be a left variable word, we immediately determine that $w_{1}(x)=x 256$ and $w_{2}(x)=x 21 x 4$. Such a conclusion would not be warranted in the event $w_{2}(x)$ is not known to be a left variable word. 
We remark that Hindman's theorem ([H1]) follows from Theorem 2.2. In this section we shall prove the following result, which strengthens Theorem 2.1 in a manner having the spirit of Theorem 2.2.

Theorem 2.3 Let $k, r \in \mathbf{N}$ and suppose $\mathcal{W}_{k}=\bigcup_{i=1}^{r} C_{i}$. Then there exists $z$, with $1 \leq z \leq r$, a variable word $w_{1}(x)$, and a sequence of left-sided variable words $\left(w_{i}(x)\right)_{i=2}^{\infty}$ such that for all $N \in \mathbf{N}$, all $b_{1}, b_{2}, \ldots, b_{N} \in \mathbf{N}$ with $1=b_{1}<b_{2}<\cdots<b_{N}$, and all $i_{1}, \cdots, i_{N} \in\{0,1, \ldots, k-1\}, w_{b_{1}}\left(i_{1}\right) w_{b_{2}}\left(i_{2}\right) \cdots w_{b_{N}}\left(i_{N}\right) \in C_{z}$.

The semigroup operation on $\mathcal{W}_{k}$ extends to its Stone-Čech compactification $\beta \mathcal{W}_{k}$ in such a way as to make $\beta \mathcal{W}_{k}$ a compact left topological semigroup, that is, a compact Hausdorff semigroup such that for fixed $f \in \beta \mathcal{W}_{k}$, the map $g \rightarrow g f$ is continuous. We exploit the algebraic structure of compact left topological semigroups in the proof of Theorem 2.3. Much of the material we need may be found in [BJM] and [HS]. Be warned, however. What we call "left topological" is referred to as "right topological" in these sources. (There is no unanimous agreement in the literature on the left-right terminology. We say left topological because the semigroup operation is continuous in the left variable.)

The following lemma of R. Ellis serves as the starting point.

Lemma 2.4 ([E, Corollary 2.10]; see also [BJM, Theorem I.3.11] or [HS, Theorem 2.5].) Any compact left topological semigroup $S$ possesses an idempotent.

Let $S$ be a compact left topological semigroup and let $J \subset S$ be non-empty. If $S J=\{s j: s \in S, j \in J\} \subset J$ then $J$ is said to be a left ideal. If $J S \subset J$ then $J$ is said to be a right ideal. If $J$ is both a left and a right ideal then we call $J$ a two-sided ideal. Any closed (left, right or two-sided) ideal, itself being a compact semigroup, contains idempotents by Lemma 2.4. If $J$ is a left ideal of $S$ that is minimal among left ideals with respect to inclusion, then we call $J$ a minimal left ideal.

The easy proof of the following lemma will be omitted.

Lemma 2.5 Let $S$ be a compact left topological semigroup.

(a) For any $x \in S, S x$ is a closed left ideal, hence if $J$ is minimal among closed left ideals then $J$ is minimal among all left ideals.

(b) Suppose $I \subset S$ is a two-sided ideal. Then $I$ contains every minimal left ideal of $S$.

(c) There exists a closed left ideal that is minimal among closed left ideals.

Taking part (c) and part (a) together, we get that minimal left ideals exist and they are closed.

Proofs of the following proposition may be found in [BJM, Theorem I.2.12], [HS, Theorem 1.38] and [M1, Proposition 2.3.1].

Proposition 2.6 Let $S$ be a compact left topological semigroup and let $\theta \in S$ be an idempotent. The following two conditions are equivalent:

(a) $\theta$ belongs to a minimal left ideal. 
(b) The only idempotent $\phi \in S$ for which $\phi \theta=\theta \phi=\phi$ is $\phi=\theta$.

An idempotent that possesses property (a), and hence property (b), of the proposition above is called a minimal idempotent. According to Lemma 2.5 (b), therefore, any two-sided ideal contains every minimal idempotent.

Lemma 2.7 (See, eg., [BJM, Corollary I.3.12] or [HS, Theorem 2.9].) Let $S$ be a compact left topological semigroup. If $R \subset S$ is a right ideal then $R$ contains a minimal right ideal.

Theorem 2.8 Let $S$ be a compact left topological semigroup. If $\theta \in S$ is a minimal idempotent and $R \subset S$ is a right ideal then there exists an idempotent $\phi \in R$ with $\theta \phi=\theta$.

Proof. By Lemma 2.7, $R$ contains a minimal right ideal $J$. Let $L$ be a minimal left ideal with $\theta \in L$. Then $L J$ is a 2 -sided ideal and hence contains $\theta$ by Lemma 2.5 (b). That is, there exists $y \in J$ and $l \in L$ such that $l y=\theta$. Since $J$ is a minimal right ideal, $y J=J$. Hence there exists $r \in J$ such that $y r=y$. Then $\theta r=(l y) r=l(y r)=l y=\theta$. Let $\phi=r \theta r$. Clearly $\phi \in R$. We now have $\phi^{2}=(r \theta r)(r \theta r)=r(\theta r) r \theta r=r(\theta r) \theta r=$ $r \theta^{2} r=r \theta r=\phi$, so $\phi$ is idempotent. Finally, $\theta \phi=\theta(r \theta r)=(\theta r)(\theta r)=\theta^{2}=\theta$, as required.

In our application of Theorem 2.8, we shall not utilize idempotence of $\phi$.

Theorem 2.9 (See [FK, Theorem 2.1]. Also [HS, Theorem 2.23] or [M1, Theorem 2.3.2].) Let $S$ be a compact left topological semigroup and let $\theta \in S$ be a minimal idempotent. If $k \in \mathbf{N}$ and $\mathcal{G} \subset S^{k}$ is a closed semigroup containing $(\theta, \theta, \cdots, \theta)$ then $(\theta, \theta, \cdots, \theta)$ is a minimal idempotent of $\mathcal{G}$.

Proof. Any idempotent in $S^{k}$ is clearly of the form $\left(\phi_{1}, \cdots, \phi_{k}\right)$, where $\phi_{i} \in S$ is idempotent. Suppose $\left(\phi_{1}, \cdots, \phi_{k}\right) \in \mathcal{G}$ is idempotent with

$$
\left(\phi_{1}, \cdots, \phi_{k}\right)(\theta, \cdots, \theta)=(\theta, \cdots, \theta)\left(\phi_{1}, \cdots, \phi_{k}\right)=\left(\phi_{1}, \cdots, \phi_{k}\right)
$$

Then $\phi_{i} \theta=\theta \phi_{i}=\phi_{i}, 1 \leq i \leq k$. But $\theta \in S$ is minimal, so $\phi_{i}=\theta, 1 \leq i \leq k$. In other words, $\left(\phi_{1}, \cdots, \phi_{k}\right)=(\theta, \cdots, \theta)$. Hence $(\theta, \cdots, \theta)$ has property (b) of Proposition 2.6, so that $(\theta, \cdots, \theta)$ is a minimal idempotent of $G$.

If $X$ is a compact Hausdorff space then it is easily shown that $X^{X}$ with the product topology forms a compact left topological semigroup under composition. If $k \in \mathbf{N},\left(X^{X}\right)^{k}$ will as well. The following easy lemma is contained in equation (2.3) of $[\mathrm{FK}]$. For another proof, see [M1, Lemma 2.3.3].

Lemma 2.10 Let $X$ be a compact space and let $k \in \mathbf{N}$. If $A, B \subset\left(X^{X}\right)^{k}$ and $A$ consists of $k$-tuples of continuous functions then $(\bar{A})(\bar{B}) \subset \overline{A B}$. 
Let $k \in \mathbf{N}$. We are finally prepared to introduce the version of the Stone-Čech compactification of $\mathcal{W}_{k}$ that we will be using. Let $X=\{0,1\}^{\mathcal{W}} \cup\{e\}$, where $e$ is the empty word. ( $e$ is an identity for $\mathcal{W}_{k}$ ). Give $X$ the product topology, so that in particular $X$ is compact. Next embed $\mathcal{W}_{k}$ in $X^{X}$ as follows: for $w \in \mathcal{W}_{k}$ let $T_{w} \in X^{X}$ be defined by $T_{w} \gamma(v)=\gamma(v w)$, where $\gamma \in X$ and $v \in \mathcal{W}_{k}$.

One may easily show that $\left\{T_{w}\right\}_{w \in \mathcal{W}_{k}}$ is a $\mathcal{W}_{k}$-action by continuous self-maps of $X$. That is, $T_{w} \circ T_{v}=T_{w v}$. We let $S$ be the closure in $X^{X}$ of $\left\{T_{w}: w \in \mathcal{W}_{k}\right\}$. That is, $S=\overline{\left\{T_{w}\right\}_{\mathcal{W}_{k}}}$; the enveloping semigroup of $\left\{T_{w}: w \in \mathcal{W}_{k}\right\}$. According to Lemma 2.10, $S$ is a subsemigroup of $\left(X^{X}\right)$ and hence itself forms a compact left topological semigroup. In fact, $S$ can be shown to be the Stone-Čech compactification of $\mathcal{W}_{k}$ (see [HS, Theorem 19.15]). We will not use that fact, however.

The following lemma will help facilitate the proof of Theorem 2.3 to follow. For $\mathbf{w}=\left(w_{1}, \cdots, w_{k}\right) \in \mathcal{W}_{k}^{k}$, let us write $T_{\mathbf{w}}=\left(T_{w_{1}}, \cdots, T_{w_{k}}\right) \in S^{k}$.

Lemma 2.11 Let $E \subset \mathcal{W}_{k}^{k}$ and suppose $\left(\phi_{0}, \phi_{1}, \ldots, \phi_{k-1}\right) \in \overline{\left\{T_{\mathbf{w}}: \mathbf{w} \in E\right\}} \subset S^{k}$. For any $\gamma_{1}, \ldots, \gamma_{m} \in X$ and any $a_{1}, \ldots, a_{m} \in \mathcal{W}_{k}$ there exists $\left(w_{0}, w_{1}, \ldots, w_{k-1}\right) \in E$ such that for all $r, 1 \leq r \leq m$, and all $j \in\{0,1, \ldots, k-1\}, \gamma_{r}\left(a_{r} w_{j}\right)=\phi_{j} \gamma_{r}\left(a_{r}\right)$.

Proof. $U=\left\{\left(\theta_{1}, \ldots, \theta_{k}\right) \in S^{k}: \theta_{j} \gamma_{r}\left(a_{r}\right)=\phi_{j} \gamma_{r}\left(a_{r}\right), 1 \leq r \leq m, j \in\{0,1, \ldots, k-1\}\right\}$ is an open neighborhood of $\left(\phi_{0}, \ldots, \phi_{k-1}\right)$. Simply pick $\mathbf{w}=\left(w_{0}, \ldots, w_{k-1}\right) \in E$ with $T_{\mathbf{w}} \in U$.

We are now ready to prove Theorem 2.3. The method employed is an adaptation of that used in $[\mathrm{FK}]$.

Proof of Theorem 2.3 Since $\mathcal{W}_{k}=\bigcup_{i=1}^{r} C_{i}$, we have $S=\bigcup_{i=1}^{r} \overline{\left\{T_{w}: w \in C_{i}\right\}}$. Pick a minimal idempotent $\theta \in S$ and choose $z$, with $1 \leq z \leq r$, such that $\theta \in \overline{\left\{T_{w}: w \in C_{z}\right\}}$. Put $\gamma=1_{C_{z}} \in X$. One checks that $\theta \gamma(e)=1$.

Let $\mathcal{I}^{\prime} \subset \mathcal{W}_{k}^{k}$ be the set of all $k$-tuples $(w(1), \cdots, w(k))$, where $w(x)$ is a variable word. Let $\mathcal{G}^{\prime}=\mathcal{I}^{\prime} \cup\left\{(w, \cdots, w): w \in \mathcal{W}_{k}\right\}$, and let $\mathcal{J}^{\prime}$ be the set of all $k$-tuples $(w(1), \cdots, w(k))$, where $w(x)$ is a left-sided variable word. Then $\mathcal{G}^{\prime}$ is a subsemigroup of $\mathcal{W}_{k}^{k}$ and $\mathcal{I}^{\prime}, \mathcal{J}^{\prime}$ are subsemigroups of $\mathcal{G}^{\prime}$ satisfying $\mathcal{G}^{\prime} \mathcal{I}^{\prime} \subset \mathcal{I}^{\prime}, \mathcal{I}^{\prime} \mathcal{G}^{\prime} \subset \mathcal{I}^{\prime}$ and $\mathcal{J}^{\prime} \mathcal{G}^{\prime} \subset$ $\mathcal{J}^{\prime}$.

Let now $\mathcal{G}=\overline{\left\{T_{\mathbf{w}}\right\}_{\mathcal{G}^{\prime}}} \subset S^{k}, \mathcal{I}=\overline{\left\{T_{\mathbf{w}}\right\}_{\mathcal{I}^{\prime}}} \subset \mathcal{G}$ and $\mathcal{J}=\overline{\left\{T_{\mathbf{w}}\right\}_{\mathcal{J}^{\prime}}} \subset \mathcal{G}$. By Lemma $2.10, \mathcal{G}$ is a compact left topological semigroup containing $\{(f, f, \cdots, f): f \in S\}, \mathcal{I}$ is a two-sided ideal in $\mathcal{G}$, and $\mathcal{J}$ is a closed right ideal in $\mathcal{G}$.

By Theorem $2.9,(\theta, \cdots, \theta)$ is a minimal idempotent in $\mathcal{G}$, therefore $\mathcal{I}$, being a two-sided ideal in $\mathcal{G}$, contains $(\theta, \cdots, \theta)$. Moreover, by Theorem 2.8 there exists $\left(\phi_{0}, \phi_{1}, \ldots, \phi_{k-1}\right) \in \mathcal{J}$ such that $\theta \phi_{i}=\theta, i \in\{0,1, \ldots, k-1\}$.

We now use Lemma 2.11 iteratively. Choose a variable word $w_{1}(x)$ such that

$$
\begin{aligned}
& \gamma\left(w_{1}\left(t_{1}\right)\right)=\theta \gamma(e)=1 \text { and } \\
& \phi_{j} \theta \gamma\left(w_{1}\left(t_{1}\right)\right)=\theta \phi_{j} \theta \gamma(e)=\theta \gamma(e)=1, j, t_{1} \in\{0,1, \ldots, k-1\}
\end{aligned}
$$


Next choose a left-sided variable word $u_{2}(x)$ such that

$$
\theta \gamma\left(w_{1}\left(t_{1}\right) u_{2}\left(t_{2}\right)\right)=\phi_{t_{2}} \theta \gamma\left(w_{1}\left(t_{1}\right)\right)=1, \quad t_{1}, t_{2} \in\{0,1, \ldots, k-1\}
$$

Then choose a variable word $v_{2}(x)$ such that

$$
\begin{aligned}
& \gamma\left(w_{1}\left(t_{1}\right) u_{2}\left(t_{2}\right) v_{2}\left(t_{2}\right)\right)=\theta \gamma\left(w_{1}\left(t_{1}\right) u_{2}\left(t_{2}\right)\right)=1 \text { and } \\
& \phi_{j} \theta \gamma\left(w_{1}\left(t_{1}\right) u_{2}\left(t_{2}\right) v_{2}\left(t_{2}\right)\right)=\theta \phi_{j} \theta \gamma\left(w_{1}\left(t_{1}\right) u_{2}\left(t_{2}\right)\right)=\theta \gamma\left(w_{1}\left(t_{1}\right) u_{2}\left(t_{2}\right)\right)=1
\end{aligned}
$$

for all $j, t_{1}, t_{2} \in\{0,1, \ldots, k-1\}$.

Let $w_{2}(x)=u_{2}(x) v_{2}(x)$. Then $w_{2}(x)$ is a left-sided variable word. Moreover,

$$
\gamma\left(w_{1}\left(t_{1}\right) w_{2}\left(t_{2}\right)\right)=1 \text { and } \phi_{j} \theta \gamma\left(w_{1}\left(t_{1}\right) w_{2}\left(t_{2}\right)\right)=1, j, t_{1}, t_{2} \in\{0,1, \ldots, k-1\}
$$

Suppose that left-sided variable words $w_{2}(x), w_{3}(x), \ldots, w_{m}(x)$ have been chosen so that letting

$$
\begin{gathered}
T_{m}=\left\{w_{1}(t): t \in\{0,1, \ldots, k-1\}\right\} \cup\left\{w_{1}\left(t_{0}\right) w_{s_{1}}\left(t_{1}\right) w_{s_{2}}\left(t_{2}\right) \cdots w_{s_{r}}\left(t_{r}\right): r \in \mathbf{N}\right. \\
\left.2 \leq s_{1}<s_{2}<\cdots<s_{r} \leq m, t_{1}, \ldots, t_{r} \in\{0,1, \ldots, k-1\}\right\}
\end{gathered}
$$

one has

$$
\gamma(w)=\phi_{j} \theta \gamma(w)=1, w \in T_{m}, j \in\{0,1, \ldots, k-1\} .
$$

Choose a left-sided variable word $u_{m+1}(x)$ such that for all $t \in\{0,1, \ldots, k-1\}$ and $w \in T_{M}, \theta \gamma\left(w u_{m+1}(t)\right)=\phi_{t} \theta \gamma(w)=1$. Next choose a variable word $v_{m+1}(x)$ such that for all $w \in T_{m}$ and $t, j \in\{0,1, \ldots, k-1\}$,

$$
\begin{aligned}
& \gamma\left(w u_{m+1}(t) v_{m+1}(t)\right)=\theta \gamma\left(w u_{m+1}(t)\right)=1 \text { and } \\
& \phi_{j} \theta \gamma\left(w u_{m+1}(t) v_{m+1}(t)\right)=\theta \phi_{j} \theta \gamma\left(w u_{m+1}(t)\right)=\theta \gamma\left(w u_{m+1}(t)\right)=1
\end{aligned}
$$

Let $w_{m+1}(x)=u_{m+1}(x) v_{m+1}(x)$. Then $w_{m+1}(x)$ is a left-sided variable word. If we now define $T_{m+1}$ by analogy with $T_{m}$, one may easily see that $\gamma(w)=\phi_{j} \theta \gamma(w)=1$ for all $w \in T_{m+1}$ and $j \in\{0,1, \ldots, k-1\}$. Hence the induction may proceed.

At this stage we are done, because the $T_{m}$ 's contain all the words we are looking for and they are all contained in $C_{z}$.

\section{Four open problems.}

In this section we discuss a few further extensions of the Hales-Jewett theorem and ask several related questions that we do not at the moment know the answer to. For starters, consider the following weak form of the Carlson-Simpson theorem. 
Theorem 3.1 Let $k, r \in \mathbf{N}$ and suppose $\mathcal{W}_{k}=\bigcup_{i=1}^{r} C_{i}$. Then there exists $j$, with $1 \leq j \leq r$, and a sequence of variable words $\left(w_{i}(x)\right)_{i=1}^{\infty}$ such that for all $N \in \mathbf{N}$ and all $i_{1}, \cdots, i_{N} \in\{0,1, \ldots, k-1\}, w_{1}\left(i_{1}\right) w_{2}\left(i_{2}\right) \cdots w_{N}\left(i_{N}\right) \in C_{j}$.

This theorem is just like Theorem 2.1 except none of the variable words are required to be left-sided. A polynomial version of Theorem 3.1 was given in [M2]. We'll formulate it in dimension 2 for ease of notation.

Recall that for $k, N \in \mathbf{N}, \mathcal{M}_{k}^{N}(2)$ denotes the set of $N \times N$ matrices $\left(a_{i j}\right)_{i, j=1}^{N}$, where $a_{i j} \in\{0,1, \ldots, k-1\}$ for all pairs $(i, j)$. Put $\mathcal{M}_{k}=\bigcup_{N=1}^{\infty} \mathcal{M}_{k}^{N}(2)$. Suppose we are given an increasing sequence $\left(R_{i}\right)_{i=1}^{\infty}$ of natural numbers (let $R_{0}=0$ ), and a sequence of non-empty sets $B_{i} \subset\left\{R_{i-1}+1, R_{i-1}+2, \cdots, R_{i}\right\}$. For every $(l, m) \in \mathbf{N} \times \mathbf{N}$, let $a_{l m}$ be the symbol $x_{i j}$ if $(l, m) \in B_{i} \times B_{j}$. Otherwise, let $a_{l m} \in\{0,1, \ldots, k-1\}$. Then $V\left(\left(x_{i j}\right)_{i, j=1}^{\infty}\right)=\left(a_{l m}\right)_{l, m \in \mathbf{N}}$ will be called a standard $\mathbf{N} \times \mathbf{N}$-variable matrix over $\mathcal{M}_{k}$. For fixed $m \in \mathbf{N}$, the matrix $V_{m}\left(\left(x_{i j}\right)_{i, j=1}^{m}\right)=\left(a_{l m}\right)_{l, m=1}^{R_{m}}$ is a standard $m^{2}$-variable matrix over $\mathcal{M}_{k}^{R_{m}}(2)$.

The variable matrix $V_{m}$ induces a natural injection $\left(t_{i j}\right)_{i, j=1}^{m} \rightarrow V_{m}\left(\left(t_{i j}\right)_{i, j=1}^{m}\right)$ from $\mathcal{M}_{k}^{m}$ to $\mathcal{M}_{k}^{R_{m}}$. Here $V_{m}\left(\left(t_{i j}\right)_{i, j=1}^{m}\right)$ is the $R_{m} \times R_{m}$ matrix which results by substituting $t_{i j}$ for the symbol $x_{i j}$ in the matrix $V_{m}\left(\left(x_{i j}\right)_{i, j=1}^{m}\right)=\left(a_{i j}\right)_{i, j=1}^{R_{m}}$ constructed above. Hence, the $\mathbf{N} \times \mathbf{N}$ matrix $V\left(\left(x_{i j}\right)_{i, j=1}^{\infty}\right)=\left(a_{l m}\right)_{l, m \in \mathbf{N}}$, together with the sequence $\left(R_{m}\right)_{m=1}^{\infty}$, induces such maps for all $m$; in other words, induces an injection of $\mathcal{M}_{k}$ into $\mathcal{M}_{k}$ (which takes $m \times m$ matrices to $R_{m} \times R_{m}$ matrices). We call the image of such a map an $\mathcal{M}_{k}$-ring. Specifically, the $\mathcal{M}_{k}$-ring generated by the sequence $\left(R_{m}\right)_{m=1}^{\infty}$ and the variable matrix $V\left(\left(x_{i j}\right)_{i, j=1}^{\infty}\right)=\left(a_{l m}\right)_{l, m \in \mathbf{N}}$.

Theorem 3.2 ([M2]) Let $k \in \mathbf{N}$. For any finite partition $\mathcal{M}_{k}=\bigcup_{i=1}^{r} C_{i}$, one of the cells $C_{i}$ contains an $\mathcal{M}_{k}$-ring.

In order to derive Theorem 3.1 from Theorem 3.2, consider the map $\Delta: \mathcal{M}_{k} \rightarrow$ $\mathcal{W}_{k}$ defined by $\Delta\left(\left(a_{i j}\right)_{i, j=1}^{N}\right)=a_{11} a_{22} \cdots a_{N N}$. If now $\mathcal{W}_{k}=\bigcup_{i=1}^{r} C_{i}$ then $\mathcal{M}_{k}=$ $\bigcup_{i=1}^{r} \Delta^{-1}\left(C_{i}\right)$ and for some $z, 1 \leq z \leq r, \Delta^{-1}\left(C_{z}\right)$ contains an $\mathcal{M}_{k}$-system generated by a standard $\mathbf{N} \times \mathbf{N}$-variable matrix $V\left(\left(x_{i j}\right)_{i, j=1}^{\infty}\right)=\left(a_{l m}\right)_{l, m \in \mathbf{N}}$. Letting $B_{i} \times B_{j}$ be the support of the variable $x_{i j}$, let for $i \in \mathbf{N} w_{i}(x)$ be the variable word that lies along the diagonal from $\left(R_{i-1}+1, R_{i-1}+1\right)$ to $\left(R_{i}, R_{i}\right)$, with $x$ replacing $x_{i i}$. That is, $w_{i}(x)=w_{1} w_{2} \cdots w_{s}$, where $s=R_{i}-R_{i-1}$ and for $1 \leq t \leq s$ and $l=R_{i-1}+t$, $w_{t}=a_{l l}$ if $a_{l l} \in\{0,1, \ldots, k-1\}$ and $w_{t}=x$ if $a_{l l}=x_{i i}$. One may easily check now that for all $N \in \mathbf{N}$ and all $i_{1}, i_{2}, \ldots, i_{N} \in\{0,1, \ldots, k-1\}, w_{1}\left(i_{1}\right) w_{2}\left(i_{2}\right) \cdots w_{N}\left(i_{N}\right)=$ $\Delta\left(V\left(\left(t_{i j}\right)_{i, j=1}^{N}\right)\right) \in C_{z}$. Here $t_{i j} \in\{0,1, \ldots, k-1\}$ are defined by $t_{j j}=i_{j}$ with $t_{i j}$, $i \neq j$, arbitrary.

It is also easy to see from this derivation what changes to Theorem 3.2 are required in order to obtain the stronger Theorem 2.1 as a consequence. In order for $w_{i}(x)$ to be a left-sided variable word, $i \geq 2$, it must be the case that the point $\left(R_{i-1}+1, R_{i-1}+1\right)$ lies in the support of $x_{i i}$. That is, $R_{i-1}+1 \in B_{i}$. An $\mathcal{M}_{k}$-system that meets this requirement for $i \geq 2$ will be called special. 
Question 3.3 Is it that case that for $k \in \mathbf{N}$ and any finite partition of $\mathcal{M}_{k}$, some cell contains a special $\mathcal{M}_{k}$-system?

One can easily formulate a version of Theorem 3.2 that would extend Theorem 2.2 as well. Let $0=R_{0}<R_{1}<\cdots<R_{N} \in \mathbf{N}$ and let $V=\left(a_{i j}\right)_{i, j=1}^{R_{N}} \in \mathcal{M}_{k}^{R_{N}}(2)$. Let $\emptyset \neq \alpha \subset\{1,2, \ldots, N\}$. We define the $\alpha$-collapse of $V$ with respect to $\left(R_{1}, \ldots, R_{N}\right)$ to be the $T \times T$ matrix, where $T=\sum_{i \in \alpha}\left(R_{i}-R_{i-1}\right)$, that is obtained by removing the rows and columns in positions $\bigcup_{i \in\{1, \ldots, N\} \backslash \alpha}\left\{R_{i-1}+1, \ldots, R_{i}\right\}$ from $V$ and "collapsing" together what is left.

This is probably best demonstrated by example. In this example, $N=3, R_{1}=2$, $R_{2}=4$ and $R_{3}=5$, while $\alpha=\{1,3\}$.

$$
\left(\begin{array}{lllll}
0 & 1 & 2 & 3 & 1 \\
0 & 3 & 1 & 2 & 1 \\
1 & 2 & 1 & 1 & 0 \\
1 & 0 & 3 & 3 & 1 \\
2 & 2 & 2 & 0 & 3
\end{array}\right) \rightarrow\left(\begin{array}{lllll}
0 & 1 & * & * & 1 \\
0 & 3 & * & * & 1 \\
* & * & * & * & * \\
* & * & * & * & * \\
2 & 2 & * & * & 3
\end{array}\right) \rightarrow\left(\begin{array}{lll}
0 & 1 & 1 \\
0 & 3 & 1 \\
2 & 2 & 3
\end{array}\right)
$$

Of course, this collapsing notion may be formalized, as well. Write $\alpha=\left\{a_{1}, \ldots a_{s}\right\}$ where $a_{1}<a_{2}<\cdots<a_{s}$. Put $a_{0}=0 .\left(b_{i j}\right)_{i, j=1}^{T}$ is the $\alpha$-collapse of $V$, where if $(m, n) \in T \times T$, and $i, j, b$ and $c$ are chosen with $0 \leq i, j<s, b \in\left\{1, \ldots, R_{a_{i+1}}-\right.$ $\left.R_{a_{i}}\right\}$, and $c \in\left\{1, \ldots, R_{a_{j+1}}-R_{a_{j}}\right\}$, such that $m=\sum_{t=0}^{i}\left(R_{a_{t}}-R_{a_{t}-1}\right)+b$ and $n=\sum_{t=0}^{j}\left(R_{a_{t}}-R_{a_{t}-1}\right)+c$, then $b_{m n}=a_{x y}$, where $x=R_{a_{i}}+b$ and $y=R_{a_{j}}+c$.

A collapsible $\mathcal{M}_{k^{-}}$system consists of an $\mathcal{M}_{k}$-system $\mathcal{N}$ generated by an increasing sequence $\left(R_{m}\right)_{m=1}^{\infty} \subset \mathbf{N}$ and a standard $\mathbf{N} \times \mathbf{N}$-variable matrix $V\left(\left(x_{i j}\right)_{i, j=1}^{\infty}\right)$, together with all the $\alpha$-collapses with respect to $\left(R_{1}, \ldots, R_{m}\right)$ of every $R_{m} \times R_{m}$ matrix in $\mathcal{N}$, for all $m \in \mathbf{N}$ and $\emptyset \neq \alpha \subset\{1, \ldots, m\}$.

Question 3.4 Is it that case that for $k \in \mathbf{N}$ and any finite partition of $\mathcal{M}_{k}$, some cell contains a collapsible $\mathcal{M}_{k}$-system?

We may combine elements of Questions 3.3 and 3.4 to formulate a version of Theorem 3.2 that would extend Theorem 2.3, also. A special $\mathcal{M}_{k}$-system, together with all of it's $\alpha$-collapses for which $1 \in \alpha$, will be called a special collapsible $\mathcal{M}_{k}{ }^{-}$ system. (One should note however that a special collapsible system need not be a collapsible system.)

Question 3.5 Is it that case that for $k \in \mathbf{N}$ and any finite partition of $\mathcal{M}_{k}$, some cell contains a special collapsible $\mathcal{M}_{k}$-system?

Again, we remind the reader that we have formulated Questions 3.3-5 in two dimensions for convenience only. We suspect that the obvious versions of each for arbitrary finite dimension are all true.

We wrap things up with two final questions dealing with possible infinitary extensions of Theorem 1.7. 
Given $k, n \in \mathbf{N}$ and an $\mathcal{M}_{k}$-ring $\mathcal{N}$ generated by a sequence $\left(R_{m}\right)_{m=1}^{\infty}$ and a standard $\mathbf{N} \times \mathbf{N}$-variable matrix $V\left(\left(x_{i j}\right)_{i, j=1}^{\infty}\right)=\left(a_{l m}\right)_{l, m \in \mathbf{N}}$, denote by $\mathcal{N}[n]$ the set of standard $n^{2}$-variable matrices that are submatrices of $V_{m}\left(\left(x_{i j}\right)_{i, j=1}^{m}\right)$ for some $m$.

Question 3.6 Let $k, r, n \in \mathbf{N}$. Supposing $\mathcal{M}_{k}[n]=\bigcup_{i=1}^{r} C_{i}$, is it the case that there must exist $i, 1 \leq i \leq r$, and an $\mathcal{M}_{k}$-ring $\mathcal{N}$ such that $\mathcal{N}[n] \subset C_{i}$ ?

The one-dimensional analogue of Question 3.6 is precisely [FK, Theorem 3.1].

It is possible to go even further. As just one example, Bergelson, A. Blass and Hindman have proved a theorem [BBH, Theorem 7.1] which stands in relation to [FK, Theorem 3.1] precisely as Theorem 2.2 stands in relation to Theorem 3.1. A two dimensional version of their result (dealing with standard variable words over collapsible systems) would stand in a similar relation to Question 3.6. We leave formulation of this and other conjectures along these lines to the reader.

\section{References}

$[\mathrm{BBH}]$ V. Bergelson, A. Blass and N. Hindman, Partition theorems for spaces of variable words, Proc. London Math. Soc. (3) 68 (1994), 449-476.

[BL] V. Bergelson and A. Leibman, Set polynomials and a polynomial extension of Hales-Jewett theorem. Ann. Math. (2) 150 (1999), 33-75.

[BJM] J. Berglund, H. Junghenn and P. Milnes, Analysis on Semigroups, Wiley, N.Y., 1989.

[C] T. Carlson, Some unifying principles in Ramsey theory, Discrete Math. 68 (1988), 117-169.

[CS] T. Carlson and S. Simpson, A dual form of Ramsey's theorem, Adv. in Math. $\mathbf{5 3}$ (1984) 265-290.

[E] R. Ellis, Lectures on topological dynamics, Benjamin, New York, 1969.

[FK] H. Furstenberg and Y. Katznelson, Idempotents in compact semigroups and Ramsey theory, Israel J. Math. 68 (1989), 257-270.

[GLR] R. Graham, K. Leeb, B. Rothschild, Ramsey's theorem for a class of categories, Adv. Math. 8 (1972), 417-433.

[HJ] A.W. Hales and R.I. Jewett, Regularity and positional games, Trans. AMS 106 (1963), 222-229.

[H1] N. Hindman, Finite sums from sequences within cells of a partition of $\mathbf{N}, J$. Combinatorial Theory (Series A) 17 (1974) 1-11.

[HS] N. Hindman and D. Strauss, Algebra in the Stone-Čech compactification-Theory and Applications, de Gruyter, Berlin, 1998.

[M1] R. McCutcheon, Elemental Methods in Ergodic Ramsey Theory, L. Notes in Math. 1722, Springer, Berlin, 1999. 
[M2] R. McCutcheon, An infinitary version of the polynomial Hales-Jewett theorem, Israel J. Math. To appear.

[Mi] K. Milliken, Ramsey's Theorem with sums or unions, J. Combinatorial Theory (Series A) 18 (1975) 276-290.

[T] A. Taylor, A canonical partition relation for finite subsets of $\omega, J$. Combinatorial Theory (Series A) 21 (1976), 137-146.

Department of Mathematics

University of Maryland

College Park, MD 20742 U.S.A. 